\title{
A NOTE ON GROUP MATRICES ${ }^{1}$
}

\section{OLGA TAUSSKY}

Let $G$ be a finite group of order $n$ with elements $P, Q, \cdots$. Let $x_{P}, x_{Q}, \cdots$ be $n$ indeterminates assumed in 1-1 correspondence with the elements of $G$. "The" group matrix corresponding to $G$ is then the $n \times n$ matrix

$$
\left(x_{P Q^{-1}}\right),
$$$$
P, Q \in G \text {. }
$$

If numerical values are given to the $x$ then we call the resulting matrix "a" group matrix.

This note gives a new characterization of hamiltonian groups by means of group matrices.

A matrix $A$ of complex numbers is called normal if $A A^{*}=A^{*} A$ where $A^{*}$ is the transposed, complex conjugate of $A$. If the matrix also contains indeterminates we introduce formally their conjugates and define normality as before. If the indeterminates are considered identical with their conjugates they are called real.

TheOREM. Let $M$ be the group matrix corresponding to the qroup $G$ and assume that the indeterminates $x$ are real. Then $M$ is a normal matrix if and only if $G$ is an abelian or a hamiltonian 2-group. If the $x$ are complex then $M$ is normal if and only if $G$ is an abelian group.

Proof. It is clear that $M$ is normal if and only if

$$
\sum_{X \in G} x_{P X^{-1}} \bar{x}_{Q X^{-1}}=\sum_{X \in G} \bar{x}_{X P^{-1}} x_{X Q^{-1}}, \quad \text { for all } P, Q \in G .
$$

If the $x$ are assumed real this is possible only if to every $P, Q, X$ there is a $Y \in G$ such that one of the two pairs of relations holds:

$$
\begin{aligned}
& P X^{-1}=Y Q^{-1}, \\
& Q X^{-1}=Y P^{-1}, \\
& P X^{-1}=Y P^{-1}, \\
& Q X^{-1}=Y Q^{-1} .
\end{aligned}
$$

Put now $P=1$, the unit element of $G$, then (i) implies that $X Q=Q X$, while (ii) implies that $Q X=X Q^{-1}$ so that $Q^{-1} X^{-1} Q X=Q^{-2}$ which gives $X^{-1} Q^{-1} X Q=Q^{2}$. Interchanging the role of $Q$ and $X$, we obtain

Received by the editors January 18, 1955.

1 This work was supported in part by the Office of Naval Research. 
that either $X$ and $Q$ commute or $X^{-1} Q^{-1} X Q=X^{-2}\left(=Q^{2}\right)$. Since $X, Q$ are arbitrary elements of the group, we conclude that any two elements $X, Q$ of $G$ either commute or satisfy the relation

$$
X^{2}=Q^{-2} \text {. }
$$

If $X, Q$ do not commute also $X, Q^{-1}$ do not commute, hence we conclude that $Q^{4}=1, X^{4}=1$. Similarly we conclude that $X^{2}=(X Q)^{2}$. This implies that $G$ is either abelian or a hamiltonian 2-group (see [1]).

If the $x$ are complex, then only the possibility (i) remains which leads to abelian groups.

We now show that the group matrix is actually normal for the groups mentioned. In the real case we have to show that to every $P, Q, X \in G$ an element $Y \in G$ exists such that either (i) or (ii) holds, i.e. either

$$
P X^{-1} Q P^{-1} X Q^{-1}=1
$$

or

$$
P X^{-1} P Q^{-1} X Q^{-1}=1 .
$$

We have to study four cases:

A. $X$ commutes with $P Q^{-1}$, but $P$ does not commute with $Q$.

B. $X$ does not commute with $P Q^{-1}$, but $P$ commutes with $Q$.

C. $X$ does not commute with $P Q^{-1}$ and $P$ does not commute with $Q$.

D. $X$ commutes with $P Q^{-1}$ and $P$ commutes with $Q$.

In cases A, B relation (iv) is satisfied, in cases $C, D$ relation (iii) is satisfied. This is shown as follows:

A. From the first assumption of $\mathrm{A}$ we have:

$$
P X^{-1} P Q^{-1} X Q^{-1}=P^{2} Q^{-2}=1
$$

since $P, Q$ do not commute and are elements of a hamiltonian group.

B. Since $X^{-1}$ does not commute with $P Q^{-1}$ we have $X^{-1} P Q^{-1}$ $=P Q^{-1} X^{-1}\left(Q P^{-1}\right)^{2}$ where $\left(Q P^{-1}\right)^{2}$ lies in the center of the hamiltonian group. Hence

$$
\begin{aligned}
P X^{-1} P Q^{-1} X Q^{-1} & =P P Q^{-1} X^{-1}\left(Q P^{-1} Q P^{-1}\right) X Q^{-1} \\
& =P Q P^{-1} Q^{-1}=1 \quad \text { by assumption. }
\end{aligned}
$$

C. Since $X$ does not commute with $Q P^{-1}$ we have $X^{-1} Q P^{-1}$ $=Q P^{-1} X^{-1}\left(P Q^{-1}\right)^{2}$ where $\left(P Q^{-1}\right)^{2}$ lies in the center of the group. Hence 


$$
\begin{aligned}
P X^{-1} Q P^{-1} X Q^{-1} & =P Q P^{-1} X^{-1}\left(P Q^{-1} P Q^{-1}\right) X Q^{-1} \\
& =P^{2} Q^{-2}=1 \quad \text { by assumption. }
\end{aligned}
$$

D. From the assumptions it follows that

$$
P X^{-1} Q P^{-1} X Q^{-1}=1 \text {. }
$$

In the complex case it follows immediately that relation (i) holds for all abelian groups.

Instead of using indeterminates $\mathrm{Ky}$ Fan suggested the use of the alternative definition for group matrices

$$
M_{f}=\left(f\left(P Q^{-1}\right)\right)
$$

where $f$ is a real-valued or complex-valued function defined on $G$. It can then be shown that the theorem can be replaced by the following result:

(i) In order that a finite group $G$ be either abelian or hamiltonian it is necessary and sufficient that for every two-valued function $f$ on $G$ taking values 0 and 1 only, the group matrix $M_{f}$ of $G$ corresponding to $f$ is a normal matrix.

(ii) In order that a finite group $G$ be abelian it is necessary and sufficient that for every three-valued function $f$ on $G$ taking values 0,1 and $i$ only, the group matrix $M_{f}$ of $G$ corresponding to $f$ is a normal matrix.

Finally, writing $f^{*}(P)=\bar{f}\left(P^{-1}\right)$ for every $P \in G$ it can be shown that normality of $M_{f}$ is equivalent with the condition

$$
\sum_{Q \in G} f\left(P Q^{-1}\right) f^{*}(Q)=\sum_{Q \in G} f^{*}\left(P Q^{-1}\right) f(Q)
$$

for all $P \in G$.

\section{BIBLIOGRAPHY}

1. R. Dedekind, Über Gruppen, deren sämtliche Teiler Normalteiler sind, Math. Ann. vol. 48 (1897) pp. 548-561 or Gesammehte mathematische Werke, II, Braunschweig, 1931, vol. 2, pp. 87-102.

National Bureau of Standards 